\title{
Innate immune signaling and regulation in cancer immunotherapy
}

\author{
Leticia Corrales $^{1, *}$, Vyara Matson ${ }^{1, *}$, Blake Flood ${ }^{1}$, Stefani Spranger ${ }^{1}$, Thomas F Gajewski ${ }^{1,2}$ \\ ${ }^{1}$ Department of Pathology, The University of Chicago, 5841 S. Maryland Ave, MC2115, Chicago, IL 60637, USA; ${ }^{2}$ Department of \\ Medicine, Section of Hematology/Oncology, The University of Chicago, Chicago, IL, USA
}

A pre-existing $T$ cell-inflamed tumor microenvironment has prognostic utility and also can be predictive for response to contemporary cancer immunotherapies. The generation of a spontaneous $T$ cell response against tumor-associated antigens depends on innate immune activation, which drives type I interferon (IFN) production. Recent work has revealed a major role for the STING pathway of cytosolic DNA sensing in this process. This cascade of events contributes to the activation of Batf3-lineage dendritic cells (DCs), which appear to be central to anti-tumor immunity. Non-T cell-inflamed tumors lack chemokines for Batf3 DC recruitment, have few Batf3 DCs, and lack a type I IFN gene signature, suggesting that failed innate immune activation may be the ultimate cause for lack of spontaneous $T$ cell activation and accumulation. With this information in hand, new strategies for triggering innate immune activation and Batf3 DC recruitment are being developed, including novel STING agonists for de novo immune priming. Ultimately, the successful development of effective innate immune activators should expand the fraction of patients that can respond to immunotherapies, such as with checkpoint blockade antibodies.

Keywords: innate immune signaling; cancer immunotherapy; dendritic cells; STING

Cell Research (2017) 27:96-108. doi:10.1038/cr.2016.149; published online 16 December 2016

\section{Introduction}

The immune system recognizes and interacts with growing tumors and immune evasion is now accepted as the seventh hallmark of cancer [1,2]. A critical recent observation is that spontaneous priming of an adaptive immune response against tumor-associated antigens is a frequent occurrence. The initial innate immune sensing of tumors results in recruitment, activation, and clonal expansion of tumor antigen-specific $\mathrm{CD} 8^{+} \mathrm{T}$ cells, which have the potential to kill cognate tumor cells. In fact, tumor-infiltrating $\mathrm{CD} 8^{+} \mathrm{T}$ cells have been detected in subsets of patients with various cancers such as melanoma and carcinomas of the head and neck, breast, lung, prostate, bladder, kidney, colon, ovary, and esophagus [3]. Importantly, this $\mathrm{T}$ cell-inflamed phenotype correlates with positive treatment outcomes in these cancers and

\footnotetext{
*These two authors contributed equally to this work. Correspondence: Thomas F Gajewski

Tel: +1-773-702-4601; Fax: +1-773-702-3163

E-mail: tgajewsk@medicine.bsd.uchicago.edu
}

has been proposed as a prognostic biomarker [3-10]. For instance, the $\mathrm{CD} 8^{+} \mathrm{T}$ cell content in the core and invasive margin of colorectal tumors, a parameter termed "immunoscore", has been reported to be a better predictor of post-surgery disease-free and overall survival than the standard TNM staging $[11,12]$. Thus, an adaptive anti-tumor immune response is spontaneously generated in a subset of patients, although without therapeutic intervention it appears to be insufficient for complete tumor rejection. One explanation for the failure of complete tumor elimination by spontaneous immune responses is the observation that tumor-infiltrating lymphocytes (TILs) become dysfunctional via dominant suppressive mechanisms in the tumor microenvironment $[13,14]$. Among these mechanisms are the induction and recruitment of immunoregulatory cells, as well as the upregulation of inhibitory pathways, such as those initiated by the checkpoint receptors cytotoxic T-lymphocyte-associated protein 4 (CTLA-4) and programmed cell death protein 1 (PD-1) expressed on TILs. Antibody-mediated blockade of CTLA-4 and PD-1 or its ligand, PD-L1, has yielded remarkable treatment outcomes in metastatic melanoma and also non-small cell lung cancer, kidney cancer, 
bladder cancer, and Hodgkin's lymphoma [15-22]. Thus, in patients with $\mathrm{T}$ cell-inflamed tumors, elimination of selected suppressive mechanisms in the tumor microenvironment and reactivation of the dysfunctional $\mathrm{T}$ cells leads to improvement of tumor control. Despite these positive advances, only a fraction of cancer patients within each tumor type exhibits a spontaneous T cell-inflamed tumor phenotype and clinical response to these checkpoint blockade agents. Thus, continued investigation is necessary to identify new strategies for expanding immunotherapy efficacy to a larger fraction of patients.

Several key differences have been identified between the $\mathrm{T}$ cell-inflamed and -non-inflamed cancer phenotypes. High numbers of TILs correlate with increased expression of multiple chemokines capable of recruiting effector T cells, including CCL2, CCL3, CCL4, CCL5, CXCL9, and CXCL10 [23]. Recent work has indicated that the most critical chemokines of them are CXCL9 and CXCL10, which are recognized by CXCR3 on effector $\mathrm{CD}^{+} \mathrm{T}$ cells [24]. Interestingly, recent mouse model data has indicated that the major source of these chemokines is the subset of dendritic cells (DCs) characterized by the basic leucine zipper transcription factor ATF-like 3 (Batf3), which in the mouse express surface CD103 or CD8 $\alpha$. This DC subset appears to play a central role in orchestrating anti-tumor $\mathrm{T}$ cell responses, both in the priming phase and at the effector phase (Figure 1).

\section{The central role for type I IFNs and Batf3 DCs}

A functionally relevant characteristic of T cell-inflamed tumors is the expression of type I IFN and IFN-inducible genes [23]. In various cancers, there is a positive correlation between the expression of type I IFN and the presence of $\mathrm{T}$ cell markers in the tumor microenvironment. The type I IFN family consists of genes encoding multiple IFN- $\alpha$ subtypes, one IFN- $\beta$, as well as the less-studied IFN- $\varepsilon,-\kappa,-\tau$, and - $\omega$ subtypes. All type I IFNs signal through a heterodimer receptor composed of one interferon alpha receptor 1 (IFNAR1) chain and one IFNAR2 chain. Ligation of the receptor causes recruitment and phosphorylation of signal transducer and activator of transcription 1 (STAT1) and STAT2. The phosphorylated STAT1 and STAT2 in complex with interferon regulatory factor 9 (IRF9) translocate to the nucleus and activate the transcription of interferon-stimulated genes by binding to IFN stimulatory response elements in their promoters [25]. In addition to a well-documented involvement of type I IFNs in anti-viral immunity and responses to intracellular pathogens, other studies have revealed a beneficial role of type I IFNs in the control of tumor growth [26-29]. Importantly, Dunn et al. [30] demonstrated that type I IFN-induced signaling in hematopoietic cells was required for the rejection of immunogenic tumors. Further studies showed that mice deficient in IFNAR or STAT1 failed to induce robust priming and expansion of anti-tumor $\mathrm{CD} 8^{+} \mathrm{T}$ cells in vivo [31]. This was not due to a $\mathrm{CD} 8^{+} \mathrm{T}$ cell-intrinsic defect, because ex vivo stimulation of $I F N A R^{-/-} \mathrm{CD}^{+} \mathrm{T}$ cells resulted in effector functions comparable to wild-type $\mathrm{CD} 8^{+} \mathrm{T}$ cells. Therefore, a defect in the innate immune cell compartment upstream of $\mathrm{CD}^{+} \mathrm{T}$ cells in IFNAR ${ }^{-/-}$and $S T A T 1^{--}$ mice is the likely culprit. Interestingly, mice with a deletion of IFNAR in CD11 $\mathrm{c}^{+}$DCs were unable to reject a model of immunogenic sarcoma [32]. Furthermore, $I F N A R^{-/-}$or $S T A T 1^{-/-}$mice exhibit a defect in the recruitment of $\mathrm{CD} 8 \alpha^{+}$DCs to the tumors [31] and IFNAR ${ }^{-/-}$ $\mathrm{CD} 8 \alpha^{+}$DCs showed impairment in cross-presentation of tumor-derived antigens to $\mathrm{CD} 8^{+} \mathrm{T}$ cells in vivo [32].

Cross-presentation, a process of internalization of cell-associated antigens and their presentation in complex with class I MHC to $\mathrm{CD} 8^{+} \mathrm{T}$ cells, is perhaps the most important functional characteristic of the CD8 $\alpha^{+}$ subset of classical DCs (cDCs) [33, 34]. In mice, CD $8 \alpha^{+}$ DCs reside in secondary lymphoid tissues and share a common differentiation pathway, cross-presentation ability, and other similarities with $\mathrm{CD} 103^{+} \mathrm{cDCs}$ found in peripheral non-lymphoid tissues $[35,36]$. Both subsets are developmentally dependent on the transcription factors IRF8 and Batf3 [37-39] and are collectively referred to as Batf3 DCs. The pivotal role of Batf3 DCs in anti-tumor immunity is evident in $\mathrm{Batf}^{-/-}$mice, which fail to reject immunogenic tumors. Using mixed bone marrow chimeras and conditional knockout models, two independent experimental approaches conclusively demonstrated that type I IFN signaling in Batf3 DCs is required for the immune control of tumor growth [31, 32]. Notably, during infection of $\mathrm{Batf}^{-/-}$mice with intracellular bacteria, Batf1 and Batf2 can interact with IRF8 and provide a compensatory restoration of the abundance and functions of Batf3 DCs via a signaling mechanism dependent on IL-12 and IFN- $\gamma$, but independent of T cells or B cells [40]. Furthermore, pre-treatment with IL12 restores the ability of $\mathrm{Batf}^{-/-}$mice to cross-prime anti-tumor $\mathrm{CD}^{+} \mathrm{T}$ cells and to reject immunogenic tumors, which appears largely due to the restoration of Batf3 DC function [40]. Although human DCs do not express CD8 $\alpha$, genome-wide expression profiling suggests that the human equivalent of murine Batf3 DCs is a population expressing blood dendritic cell antigen 3 (BDCA3, also known as CD141) identified in peripheral blood [41]. Like the murine Batf3 DCs, human CD $141^{+}$DCs express the transcription factors IRF8 and Batf3; express C-type lectin domain family 9 member A (Clec9A, also known 


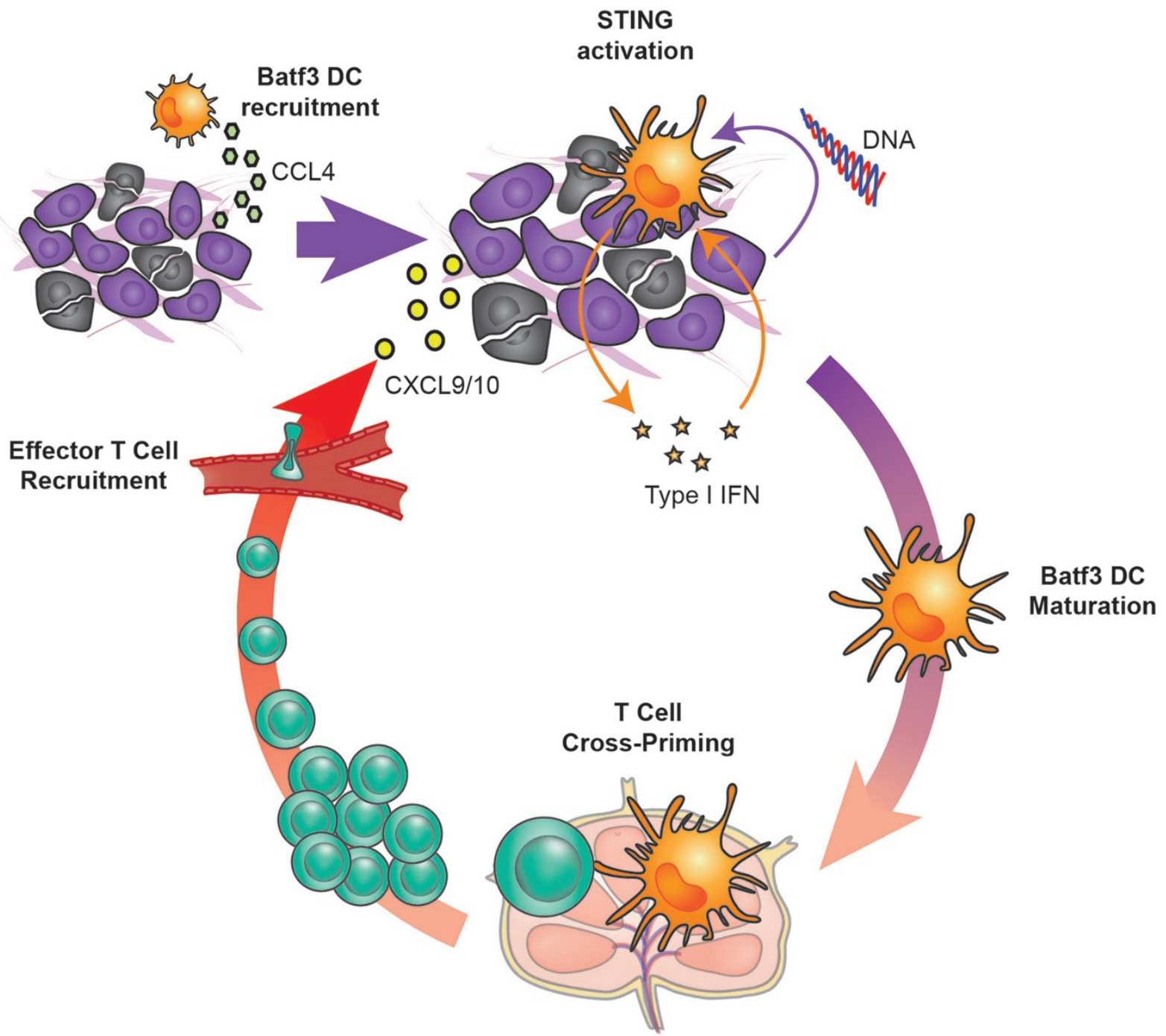

Figure $1 \mathrm{~A}$ central role of Batf3 DCs and the STING pathway in orchestrating anti-tumor T cell responses. Tumor cells displaying a T cell-inflamed phenotype produce chemokines, such as CCL4, which recruit Batf3 DCs. Tumor cell turnover and perhaps death results in the liberation of DNA and other DAMPs that gain access to infiltrating APCs, including DCs. Cytosolic DNA in APCs activates the STING pathway, resulting in the secretion of type I IFNs and also of the chemokines CXCL9 and CXCL10. Tumor endothelial cells can also contribute to the production of type I IFNs. This cytokine subsequently facilitates the activation of tumor-infiltrating Batf3 DCs, which take up tumor-associated antigens and migrate via the lymphatics to the tumor-draining lymph node, where they cross-prime tumor-specific CD8 ${ }^{+} \mathrm{T}$ cells. The activated $\mathrm{CD} 8^{+} \mathrm{T}$ cells undergo clonal expansion in the tumor-draining lymph node and traffic via the bloodstream. They are recruited back to the tumor microenvironment under the influence of CXCL9 and CXCL10 also released by Batf3 DCs in the tumor microenvironment. Dynamic movement of infiltrated $\mathrm{CD}^{+}$effector $\mathrm{T}$ cells results in direct contact with tumor cells, which can lead to tumor cell death. If tumor cells are not completely eliminated, then immune inhibitory pathways suppress $T$ cell activation as part of a negative feedback loop.

as DNGR1), which potentiates cross-presentation of antigens derived from necrotic cells [42]; and secrete IL-12 leading to $\mathrm{T}_{\mathrm{H}} 1$ polarization $[43,44]$.

The role of type I IFNs is critical in the early stages of anti-tumor immune response, because a blocking antibody against this cytokine prevented rejection of a transplantable immunogenic tumor if administered within the first 4-6 days of tumor growth, but had no effect at later time points [32]. Indeed, at this early stage, type I IFN is produced by $\mathrm{CD} 11 \mathrm{c}^{+}$cells in the tumor-draining lymph node in the B16 melanoma model [31]. In fact, in this model, type I IFN is also induced in tumor-in- 
filtrating DCs and myeloid cells as early as day 1 after tumor inoculation [45]. Type I IFNs are also expressed by leukocytes in the tumor and tumor-draining lymph node in an autochthonous melanoma model driven by melanocyte-specific activating mutation in $B R A F$ and deletion of PTEN [45]. Recently, it was demonstrated that $\mathrm{CD} 103^{+}$DCs are uniquely capable of transporting tumor-derived antigens to the tumor-draining lymph node in a CCR7-dependent manner, where they prime antigen-specific $\mathrm{CD} 8^{+} \mathrm{T}$ cells [46]. This process was augmented in an IFNAR-dependent manner, revealed by a combination treatment of tumor-bearing mice with Fms-related tyrosine kinase 3 ligand (Flt3L) and intratumoral injection of polyinosinic:polycytidylic acid (poly I:C). The improvement of tumor control with this treatment was attributed to Flt3L-mediated expansion of the tumor-infiltrating $\mathrm{CD} 103^{+} \mathrm{DCs}$ and their activation by poly I:C via the toll-like receptor 3 (TLR3)-TIR-domain containing adapter-inducing IFN- $\beta$ (TRIF) pathway [46]. Collectively, these findings support a model in which type I IFN production in the tumor facilitates antigen cross-presentation by Batf3 DCs, which then migrate via the lymphatics to the tumor-draining lymph nodes, where they cross-prime naive $\mathrm{CD} 8^{+} \mathrm{T}$ cells.

In further support of a pivotal role of Batf3 DCs in anti-tumor immunity, a recent study demonstrated that $\mathrm{CD}_{103}{ }^{+} \mathrm{DCs}$ in the lung internalize membrane-bound cytoplasmic material (which they termed "cytoplast") released in the lung vasculature by circulating tumor cells [47]. As early as $72 \mathrm{~h}$ after injection of OVA-expressing tumor cells, these "cytoblast"-loaded CD103 ${ }^{+}$DCs were detected in mediastinal lymph nodes, clustering together with adoptively transferred OT-I T cells, which exhibited a morphology consistent with cell activation. The ability of these $\mathrm{CD} 103^{+}$DCs to activate OT-I T cells was conclusively confirmed ex vivo and using bone marrow chimeras [47].

In addition to the classical notion that the site for activation of tumor-specific $\mathrm{CD} 8^{+} \mathrm{T}$ cells is the tumor-draining lymph node, cross-priming of naive $\mathrm{CD} 8^{+} \mathrm{T}$ cells has also been reported to occur in the tumor microenvironment [48]. This appears to be due to the presence in the tumors of vasculature resembling the high endothelial venules characteristic of lymph nodes. Through the expression of peripheral node addressins (PNAd) and CCL21 on the luminal endothelial surface, these blood vessels supported the extravasation of naive $\mathrm{CD} 8^{+} \mathrm{T}$ cells, which were subsequently activated in the tumor $[48,49]$. Thus, intratumoral expression of type I IFNs may even prompt tumor-infiltrating Batf3 DCs to cross-prime naive $\mathrm{CD} 8^{+}$ $\mathrm{T}$ cells in the tumor, bypassing the need for migration to the draining lymph nodes.

\section{The STING pathway and innate immune sensing of tumors}

A major conundrum had been how antigen-presenting cells (APCs) in the tumor microenvironment could productively cross-present tumor antigens to $\mathrm{T}$ cells in the absence of pathogen-derived factors. Now it is well established that under sterile conditions, pattern recognition receptors (PRRs) can sometimes be activated by endogenous factors [50], bridging innate immune activation to adaptive immunity. However, in the tumor setting the nature of such tumor-related factors and the main pathways activated during carcinogenesis have only recently begun to be elucidated. The association between a type I IFN gene signature and T cell infiltration in human cancers as well as mouse tumor models allowed a focus on innate signaling pathways capable of inducing type I IFNs [23, 31, 32]. As described above, deficiency in IFNAR or the downstream transcription factor STAT1 in host cells resulted in impaired priming of T cells against tumor-associated antigens [31, 32]. Production of IFN- $\beta$ was detected in response to tumor challenge in tumor-draining lymph nodes, predominantly by $\mathrm{CD} 11 \mathrm{c}^{+}$ cells consistent with DCs as a major source. Downstream type I IFN signaling was specifically required in Batf3 DCs for T cell priming to occur, as well as for subsequent immune-mediated tumor control. Other cells in the tumor can contribute to production of type I IFNs, including tumor endothelial cells [51].

The involvement of type I IFNs in the innate immune sensing of cancer provided a tool for probing candidate PRR pathways. Through the use of gene-targeted mice specifically deficient in defined receptors and signaling proteins, the necessity of specific pathways for spontaneous $T$ cell priming against tumor-associated antigens was pursued. Mice deficient in specific TLRs, including TLR4 or TLR9, or lacking the downstream adapters myeloid differentiation primary response gene 88 (MyD88) and/or TRIF, showed intact priming of tumor antigen-specific $\mathrm{CD}^{+} \mathrm{T}$ cells. Similarly, mice deficient in the cytosolic RNA sensing pathway via MAVS, and the purinergic receptor P2X7R that is activated by extracellular ATP [52], had intact anti-tumor immunity. However, mice lacking the adaptor molecule stimulator of interferon genes (STING, also known as TMEM173, MITA, ERIS, and MPYS) or the transcription factor IRF3, which is activated downstream of STING stimulation, showed blunted anti-tumor $\mathrm{T}$ cell priming and deficient rejection of transplantable tumors $[45,53]$. The host STING pathway has also been shown to be protective in other tumor models, including in a mouse model of colitis-associated carcinogenesis induced by azoxymethane/ 
dextran sodium sulfate [54-56] and in a glioma model caused by a sleeping beauty transposon system [57].

The STING pathway has been defined to survey the presence of cytosolic DNA. In the characterized pathway, cyclic-GMP-AMP (cGAMP) synthase (cGAS, also known as MB21D1) senses cytosolic DNA and catalyzes the synthesis of cGAMP [58]. cGAMP, the defined endogenous ligand of STING [59], then induces conformational changes in STING, which causes its subsequent trafficking from the endoplasmic reticulum to perinuclear vesicles [60]. This results in the recruitment and phosphorylation of TANK-binding kinase 1 (TBK1), which in turn phosphorylates and activates IRF3 leading to the activation of type I IFN transcription [61]. Based on this model of STING pathway activation, the presence of tumor-derived DNA has been examined in the cytosol of intratumoral APCs and does appear to be found there [45, 53]. In vitro, the delivery of tumor-derived DNA into APCs using lipofectamine was also sufficient to activate the STING pathway and elicit type I IFN production. Together, these data are consistent with the idea that STING activation in host APCs in the tumor is triggered upon successful transfer of DNA from tumor cells. However, there is also the potential to transfer cGAMP directly, which in some model systems has been reported to occur through gap junctions $[62,63]$. The detailed cell biology of STING pathway activation in the cancer context is the subject of current investigation.

Although it is now well established that activation of STING can lead to an anti-tumor T cell response and thus tumor control, chronic activation of the pathway in models of inflammation-induced carcinogenesis can in some cases promote tumorigenesis. In a model of cutaneous skin tumors induced by 7,12-dimethylbenz[a] anthracene (DMBA), STING-deficient animals showed a better outcome [64]. This was explained by the persistent generation of downstream cytokines and recruitment of phagocytes, which created an inflammatory milieu that promoted tumor development $[54,64]$. It also has been reported in the context of a non-immunogenic transplantable tumor that deficiency of STING increases tumor protection. In this model, activation of STING by DNA or STING agonists promoted tolerogenic responses by induction of indoleamine 2,3 dioxygenase (IDO), which activated Tregs to promote dominant inhibitory $\mathrm{T}$ cell regulation $[65,66]$. A recent study has linked the activation of STING and production of inflammatory cytokines to brain metastasis and chemoresistance [63]. In this case, brain metastatic cancer cells established gap junctions with astrocytes and used these channels to transfer cGAMP. Activation of the STING pathway in astrocytes led to the generation of pro-inflammatory cytokines that in a paracrine loop activated the STAT1 and NF- $\kappa \mathrm{B}$ pathways in brain metastatic cells, supporting tumor growth. These studies indicate that in some conditions STING activation might facilitate inflammation-induced carcinogenesis, thus an appropriate balance in STING pathway activation may be required for optimal anti-tumor effects.

\section{Potential role for additional innate immune sensors}

The uncovered role of the host STING pathway in the innate immune sensing of cancer provides one explanation for how anti-tumor $\mathrm{T}$ cells can become primed in the absence of pathogen-derived innate stimuli. In addition, several other molecules and pathways have been suggested to be operational in some model systems.

High mobility group box 1 (HMGB1) is a nuclear non-histone chromatin-binding protein that is released following cellular necrosis but not apoptosis [67]. It is also released following treatment with certain chemotherapies [68]. HMGB1-induced inflammation following cell death has been reported to be TLR4-dependent, suggesting that TLR4 is a receptor for HMGB1. Other studies have found that T-cell immunoglobulin and mucin-domain containing-3 (TIM-3) on the surface of tumor-associated DCs also interacts with HMGB1 and suppresses APC activation [69]. Oxidized HMGB1 purified from cell lysates seems to be suppressive, whereas the reduced form can activate immune cells $[70,71]$. Recombinant HMGB1 is not sufficient to initiate inflammation, suggesting that an unidentified interacting factor may be required [72]. Therefore, additional work is needed to understand the precise role for HMGB1 in anti-tumor immunity.

ATP is also reported to induce inflammation under specific conditions. ATP-induced inflammation depends on innate immune $\mathrm{P} 2 \mathrm{X} 7$ receptors, which require high concentrations of ATP for activation. However, immune activation has been reported to be suppressed by adenosine generated from the breakdown of ATP by CD73, implying a kinetic window for ATP-induced inflammation [73]. CD73 is present on the surface of tumor cells and regulatory immune cells, suggesting that tumors might be capable of converting this inflammatory stimulus into an immunosuppressive signal.

Tumor-derived RNA can also activate innate immunity and tumor cells appear to actively exploit this pathway for their own benefit. Tumor cells aberrantly express a number of miRNAs that are actively secreted in extracellular vesicles called exosomes. Some of these miRNAs bind to murine TLR7 and human TLR8 with sequence specificity [74]. TLR activation by these miRNAs leads to TNF- $\alpha$ and IL-6 production in vitro. In an in vivo 
model of lung metastasis formation, cells expressing these miRNAs generated more lung metastases than cells transfected with control vectors [74]. Thus miRNAs in this context seem to produce pro-metastatic inflammation.

Uncontrolled activation of innate immune pathways and generation of innate cytokines can lead to autoimmunity. Thus, cells are equipped with regulatory mechanisms that counterbalance inflammatory responses. In particular, inappropriate activation of the STING pathway and generation of type I IFNs could promote the Aicardi-Goutières syndrome [75] or systemic lupus erythematosus [76]. Several mechanisms for negative regulation of the STING pathway have been described, such as elimination of accumulated DNA by DNases [77] and post-translational modification of proteins in the pathway after stimulation [78]. Recent work has indicated another level of regulation, which is dual stimulation of two innate immune pathways by the same ligand having opposing functional consequences. In particular, cytosolic DNA can activate both the STING pathway and the absent in melanoma 2 (AIM2) inflammasome in APCs. AIM2 senses DNA and forms a heterocomplex with the adaptor protein apoptosis-associated speck-like protein (ASC). Formation of the functional inflammasome complex activates caspase- 1 that in turn acts to generate matured forms of IL-1 $\beta$ and IL-18 [79], and causes a type of cell death called pyroptosis [80]. APCs that are deficient in the AIM2 inflammasome show marked overactivation of the STING pathway [81], indicating that induction of pyroptosis by the AIM2 inflammsome normally acts to dampen STING pathway activation. This result indicates that cross-talk between different innate immune pathways can potentially lead to unexpected outcomes and offers new opportunities to target and modulate immune responses.

The complexity of intercellular interactions in the tumor microenvironment could impact the regulation of innate immune sensing pathways during the evolution of the tumor-host interaction. Many pathways appear to be capable of sensing tumor-derived factors in specific settings, but likely have varying downstream effects depending on the cellular context and the nature of the stimulus received. For example, both Tregs and suppressive myeloid cells termed "myeloid-derived suppressor cells" express CD73 that can degrade the inflammatory molecule ATP, converting it into an immunosuppressive signal adenosine. Similarly, different subsets of DCs present in the tumor (and specifically their expression of TIM-3 compared to TLR4) may regulate responses to HMGB1. Therefore, whether tumors support productive inflammation likely depends not only on tumor-intrinsic release of PRR ligands, but also the state of stromal cells that sense them.

Additional innate cell subsets also may impact the early phases of anti-tumor immunity and/or the clinical efficacy of immunotherapies in the setting of established tumors. NK cells have been reported to contribute to tumor control in some settings [82], but in established B16 tumors NK cells also have been shown to have regulatory function resulting in immune suppression, in part through PD-L1 expression [83]. Similarly, $\gamma \delta T$ cells are capable of direct tumor recognition and in some circumstances can be activated to kill tumor cells, but at the same time $\gamma \delta \mathrm{T}$ cells with regulatory function have been identified from human breast cancer tissues [84]. It also has been described that NKT cells can be either anti-tumor or tumor-promoting [85]. Until recently, little information has been available regarding innate lymphoid cell populations and anti-tumor immunity. However, a recent report in which a polyoma middle-T antigen transgenic mouse model was used to study early breast cancer development revealed that the earliest host cells accumulating in the tumor microenvironment are innate lymphoid-like cells that contribute to tumor control [86]. Overall, additional work will be needed to interrogate the functional contributions of these additional innate immune cell subsets in human cancers and also in the context of immunotherapy response versus resistance.

\section{Functional interaction with the commensal microbiota}

Another host factor that appears to impact innate immune cell activation is the composition of the commensal microbiota. The most diverse and dense microbiota resides in the gastrointestinal tract, mainly in the colon, contributing qualitatively and quantitatively to intestinal homeostasis [87]. Intestinal commensals provide structural and biological functions including nutrient digestion and drug metabolism. Importantly, commensals have evolved closely with their hosts in a symbiotic relationship, and this interaction also shapes the immune system. At the same time, the immune system exerts "inside-out" control over microbiota localization and composition [88]. Interestingly, recent studies demonstrate that gut commensals have an important impact on systemic anti-tumor immune responses. Genetically similar C57BL/6 mice from two different vendors, Jackson (JAX) and Taconic (TAC), which are known to have different gut microbiota [89], showed high versus low spontaneous T cell responses against tumors. This difference correlated with the rate of tumor growth in the two cohorts of mice [90]. Interestingly, cohousing of mice was sufficient to eliminate the difference in phenotype. Moreover, transfer 
of fecal material from JAX mice (which had superior anti-tumor immunity) to TAC mice had a therapeutic effect, increasing tumor antigen-specific $\mathrm{T}$ cell frequencies and slowing tumor growth in TAC mice. Sequencing of the gut microbiome of TAC, JAX, and TAC mice that received JAX fecal material showed that the presence of certain Bifidobacterium species was linked to the improved anti-tumor immune response. Administration of a cocktail of Bifidobacterium strains to tumor-bearing TAC mice replicated the benefit of the fecal transplant from JAX mice, and synergized with anti-PD-L1 mAb for maximal therapeutic benefit. Mechanistic studies demonstrated that DCs isolated from either JAX mice or Bifidobacterium-treated TAC mice were able to better stimulate tumor antigen-specific $\mathrm{T}$ cells in vitro. Gene expression profiling of these DCs revealed evidence for a modest pre-activation of DCs when Bifidobacterium was present, arguing for an innate immune mechanism for this improved immune activation effect [90]. This observation is similar to what has been reported in a viral infection model, in which gut bacteria induced APC "poising" [91]. In agreement, germ-free or antibiotic-treated mice, which completely lack or have a compromised commensal microbiota, showed defective innate immunity during viral infection [92, 93]. Furthermore, in the tumor context, germ-free mice and antibiotic-treated mice were resistant to anti-CTLA-4 therapy [94]. Oral transfer of B. fragilis or B. thetaiotaomicron recovered the anti-CTLA-4 therapeutic effect, demonstrating that these bacteria are sufficient to promote anti-CTLA-4 efficacy. Mechanistically, in this model anti-CTLA-4 mAb induced subclinical colitis that could favor bacterial colonization of the mucosal layer and could explain the observed increased frequencies of Bacteroides-reactive $\mathrm{CD}^{+} \mathrm{T}$ cells in melanoma patients following ipilimumab therapy. Apart from checkpoint blockade therapy, the therapeutic effects of chemotherapy with oxaliplatin or of anti-IL-10R plus CpG were facilitated by gut microbes $[95,96]$. In these contexts, the therapeutic effect mediated by the microbiota relied on the production of cytokines by myeloid cells [95], or translocation of bacteria to peripheral organs, which increased Th17 cells involved in the anti-tumor response [96]. Overall, these seminal studies demonstrate how different commensals affect the basal state of endogenous innate immune cells and could favor anti-tumor responses, as well as affect the therapeutic effect of immunotherapies and conventional anti-cancer treatment.

\section{Mechanisms of $\mathbf{T}$ cell exclusion from tumors linked to defective innate immune interactions}

Clinical responses observed to immunotherapeutic interventions have been strongly associated with the baseline presence of a $\mathrm{CD} 8^{+} \mathrm{T}$ cell infiltrate $[9,97]$. Gene expression profiling of the tumor microenvironment has revealed the presence of $\mathrm{T}$ cell transcripts, chemokines, and a type I IFN gene signature in a major subset of human cancers $[9,23,98,99]$. This T cell-inflamed tumor microenvironment gene signature has been associated with clinical response to therapeutic vaccines, the anti-CTLA-4 mAb, and more recently anti-PD- $1 \mathrm{mAb}[100$, 101]. Using immunohistochemical approaches, the presence of $\mathrm{CD}^{+} \mathrm{T}$ cells in the tumor itself or at the tumor margin has been correlated with anti-PD-1 efficacy [15]. Interestingly, on-treatment biopsies revealed the accumulation of $\mathrm{Ki} 67^{+} \mathrm{T}$ cells in responding patients, arguing that in situ proliferation of anti-tumor T cells is a major component of clinical efficacy.

The above observations have suggested that the absence of tumor-infiltrating $\mathrm{CD} 8^{+} \mathrm{T}$ cells approximates a state of resistance to checkpoint blockade immunotherapy. As such, deeper mechanistic insight is being sought to understand why a subset of tumors in some patients fails to support the priming and/or recruitment of tumor antigen-specific $\mathrm{T}$ cells. Based on the hypothesis that one source of inter-patient heterogeneity lies in the constellation of somatic changes in the tumor cells themselves, the $\mathrm{T}$ cell-inflamed tumor microenvironment gene signature has been analyzed in parallel with exome sequencing of the same tumors in a subset of 266 metastatic melanomas represented in the cancer genome atlas (TCGA). This analysis revealed evidence for preferential $\mathrm{Wnt} / \beta$-catenin activation in $48 \%$ of non-T cell-inflamed tumors [102]. Using genetically engineered mouse models for melanoma driven by active B-Raf and PTEN deletion with or without constitutive activation of $\beta$-catenin, it was found that tumors expressing $\beta$-catenin completely lost the spontaneous $\mathrm{T}$ cell infiltrate normally observed. This was attributed to a complete failure of initial priming of CD8 $\mathrm{T}$ cells against tumor-associated antigens. Because of the central function of Batf3 DCs in the initiation of anti-tumor immune responses in transplantable tumor models, a mechanism was pursued around this DC subset. Indeed, lack of $\mathrm{T}$ cell priming was found to be caused by reduced recruitment of $\mathrm{CD}_{103} /$ Batf3-driven DCs. Lack of this DC recruitment was attributed to failed production of the chemokine CCL4 by the tumor cells, which was transcriptionally repressed via $\beta$-catenin activation of ATF3 [102]. In the $\beta$-catenin-negative tumors, infiltrating DCs were found to produce IFN- $\beta$, just like in the transplantable tumor models. However, the few DCs that were detected in the $\beta$-catenin-expressing tumors failed to make IFN- $\beta$. Recently, we have confirmed that this spontaneous IFN- $\beta$ production in the genetically engineered 
tumor model is also lost in $S T I N G^{-/-}$hosts (Spranger and Gajewski, unpublished data). Together, these data suggest that the absence of $\mathrm{CD} 8^{+} \mathrm{T}$ cell infiltration in a major subset of solid tumors is likely due to lack of recruitment and activation of Batf3 DCs. Indeed, we have observed a positive correlation of markers of the Batf3 DC lineage (Batf3, IRF8, CD141) with CCL4 and also CD8 $\alpha$ within the TCGA data set across solid tumors (Spranger and Gajewski, unpublished data). Batf3 is also associated with a type I IFN gene signature [102]. Because activation of the $\mathrm{Wnt} / \beta$-catenin pathway is only observed in a subset of non-T cell-inflamed cancers, these observations suggest that other molecular mechanisms responsible for the failed recruitment and activation of Batf3 DCs likely exist in other tumor subsets.

\section{Therapeutic opportunities}

Based on the notion that recruitment and activation of Batf3 DCs is likely a cornerstone to initiate a de novo immune response against tumors, several novel therapeutic interventions can be envisioned. The most obvious strategy to consider is direct injection of activated Batf3 DCs into the tumor microenvironment. In the $B$-Raf $/ P T E N^{1-}$, $\beta$-catenin genetically engineered mouse model, this has been pursued by generating bone marrow-derived DCs using Flt3L followed by activation with the TLR3 agonist poly I:C. Injection of these DCs directly into the tumor microenvironment restored $\mathrm{T}$ cell priming, effector $\mathrm{T}$ cell recruitment, and caused partial tumor shrinkage, which was amplified with co-administration of anti-CTLA-4 and anti-PD-L1 mAbs [102]. In a related strategy, systemic administration of Flt3L to increase Batf3 DCs in the tumor microenvironment, combined with intratumoral injection of poly I:C had a similar effect [46]. These are approaches that are immediately testable in the clinic with available reagents.

A second set of strategies is focused on STING pathway activation. Preclinical exploration began with the agent 5,6-dimethylxanthenone-4-acetic acid (DMXAA), which was recently demonstrated to directly interact with murine STING [103-106]. A single intratumoral dose of DMXAA in B16 melanoma-bearing mice was sufficient to promote rejection of most tumors, which was associated with a marked augmentation in the frequency of tumor antigen-specific $\mathrm{CD}^{+} \mathrm{T}$ cells. DMXAA showed a similarly potent therapeutic effect in other transplantable mouse models of prostate cancer, breast cancer, and sarcoma. In addition, mice that completely rejected tumors were protected against a rechallenge with the same tumor cell line, implying the generation of an immunological memory [107]. Although DMXAA failed in a phase III clinical trial of non-small cell lung carcinoma [108], now it is well known that this was due to its inability to bind human STING. New agents are being developed that engage human STING. One major approach involves rational modifications of cyclic dinucleotides (CDNs) to improve efficiency, which led to the development of synthetic dithio-mixed linkage CDNs [107]. One compound (ML RR-S2 CDA or ADU-S100) binds both human and mouse STING, and showed a potent anti-tumor effect in multiple animal models [107]. A phase 1 clinical trial of ADU-S100 in patients with cutaneously accessible solid tumors and lymphomas has been initiated [109].

Other innate immune pathway activators are being tested in the clinic as single agents or in combination with other therapies, with the aim to boost anti-tumor $\mathrm{T}$ cell responses [110-112]. Similar to STING agonists, intratumoral injection of TLR agonists such as CpG-rich oligodeoxynucleotides (CpG ODN, PF-3512676) along with low-dose radiotherapy has shown clinical responses in patients with advanced non-Hodgkin's lymphoma in a phase I/II clinical study [113]. The TLR4 agonist glucopyranosyl lipid adjuvant (GLA) is being tested alone or in combination with anti-PD-1 mAb [114]. The TLR3 agonist Poly-ICLC (Hiltonol), and the TLR7/8 agonist MEDI9197, are also being tested in patients with advanced accessible solid tumors [115, 116].

Preclinical data have indicated that the efficacy of stereotactic radiation of tumors, which as part of its mechanism of action boosts anti-tumor immune responses, largely depends on the host STING pathway [117]. Radiation therapy, therefore, is in part an indirect STING agonist. Clinical trials have been initiated combining stereotactic radiation with anti-PD-1 mAb [118] in an attempt to convert non-T cell-inflamed tumors into $\mathrm{T}$ cell-inflamed tumors, which may synergize with checkpoint blockade immunotherapy.

Finally, as the molecular mechanisms in the tumor cells continue to be identified that are ultimately causal for $\mathrm{T}$ cell exclusion, new targeted therapies can be envisioned to block those pathways and enable endogenous immune responses to proceed. The first pathway identified is the $\mathrm{Wnt} / \beta$-catenin pathway in melanoma [102], and new efforts are being invested into targeting this pathway pharmacologically. Our current estimate is that this pathway is associated with $\mathrm{T}$ cell exclusion in at least 20 additional cancer types [119]. Recent work has pointed toward several additional tumor-intrinsic oncogene pathways that are associated with immune exclusion and are also ripe for targeting. These include the PTEN/PI3K pathway [120] in melanoma, the FGFR3 pathway in bladder cancer [121], and c-myc in hematologic malignancies [122]. As an exhaustive analysis of 
additional molecular pathways is collated through analysis of TCGA and other ongoing prospective genomic studies of samples in the context of immunotherapy clinical trials, it is expected that a prioritized list of candidate drug targets will emerge.

\section{Concluding remarks}

Innate immune activation plays a critical role in the spontaneous adaptive immune response against cancer. Recent work has implicated the STING pathway of cytosolic DNA sensing as a major pathway involved in this process, which is associated with $\mathrm{T}$ cell infiltration and the ability to respond to checkpoint blockade immunotherapy. Central in this cascade of events are Batf3-lineage DCs, which respond to IFN- $\beta$ produced downstream of STING activation that facilitates cross-presentation of antigens to $\mathrm{CD} 8^{+} \mathrm{T}$ cells. Knowledge of this cascade has led to novel clinical/translational approaches to trigger the STING pathway and/or promote Batf3-lineage DC recruitment and activation. Ultimately, innate immune pathway agonists might promote de novo activation and recruitment of anti-tumor $\mathrm{T}$ cells, which still may become held in check by negative regulatory pathways such as PD-1/PD-L1 interactions. Thus, one might envision combination therapies in the future, establishing a $\mathrm{T}$ cell-inflamed tumor microenvironment phenotype with innate immune activators and then blocking negative regulation with agents such as anti-PD-1 mAb. Future work should integrate a more detailed evaluation of additional innate-like cells in the tumor microenvironment, in the context of immunotherapy response and therapeutic efficacy.

\section{Acknowledgments}

LC and SS are supported by Cancer Research Institute Irvington postdoctoral fellowships. BF is supported by an NIH Medical Scientist Training Program grant T32GM007281. Work described in this review was supported in part by R01 CA181160 and R01 CA198496 from the NIH.

\section{Competing Financial Interests}

LC and TG are partly in a patent application on the role of STING agonists as a cancer therapy. The remaining authors declare no conflict of interest.

\section{References}

1 Dunn GP, Old LJ, Schreiber RD. The three Es of cancer immunoediting. Annu Rev Immunol 2004; 22:329-360.

2 Hanahan D, Weinberg RA. Hallmarks of cancer: the next generation. Cell 2011; 144:646-674.

3 Pages F, Galon J, Dieu-Nosjean MC, Tartour E, Sautes-Frid- man C, Fridman WH. Immune infiltration in human tumors: a prognostic factor that should not be ignored. Oncogene 2010; 29:1093-1102.

4 Mlecnik B, Tosolini M, Kirilovsky A, et al. Histopathologic-based prognostic factors of colorectal cancers are associated with the state of the local immune reaction. J Clin Oncol 2011; 29:610-618.

5 Azimi F, Scolyer RA, Rumcheva P, et al. Tumor-infiltrating lymphocyte grade is an independent predictor of sentinel lymph node status and survival in patients with cutaneous melanoma. J Clin Oncol 2012; 30:2678-2683.

6 Mahmoud SM, Paish EC, Powe DG, et al. Tumor-infiltrating CD8+ lymphocytes predict clinical outcome in breast cancer. J Clin Oncol 2011; 29:1949-1955.

7 Rusakiewicz S, Semeraro M, Sarabi M, et al. Immune infiltrates are prognostic factors in localized gastrointestinal stromal tumors. Cancer Res 2013; 73:3499-3510.

8 Hwang WT, Adams SF, Tahirovic E, Hagemann IS, Coukos G. Prognostic significance of tumor-infiltrating T cells in ovarian cancer: a meta-analysis. Gynecol Oncol 2012; 124:192-198.

9 Gajewski TF, Louahed J, Brichard VG. Gene signature in melanoma associated with clinical activity: a potential clue to unlock cancer immunotherapy. Cancer J 2010; 16:399-403.

10 Hamid O, Schmidt H, Nissan A, et al. A prospective phase II trial exploring the association between tumor microenvironment biomarkers and clinical activity of ipilimumab in advanced melanoma. $J$ Transl Med 2011; 9:204.

11 Pages F, Kirilovsky A, Mlecnik B, et al. In situ cytotoxic and memory $\mathrm{T}$ cells predict outcome in patients with early-stage colorectal cancer. J Clin Oncol 2009; 27:5944-5951.

12 Galon J, Mlecnik B, Bindea G, et al. Towards the introduction of the 'Immunoscore' in the classification of malignant tumours. J Pathol 2014; 232:199-209.

13 Ahmadzadeh M, Johnson LA, Heemskerk B, et al. Tumor antigen-specific CD8 $\mathrm{T}$ cells infiltrating the tumor express high levels of PD-1 and are functionally impaired. Blood 2009; 114:1537-1544.

14 Zippelius A, Batard P, Rubio-Godoy V, et al. Effector function of human tumor-specific CD8 T cells in melanoma lesions: a state of local functional tolerance. Cancer Res 2004; 64:28652873.

15 Tumeh PC, Harview CL, Yearley JH, et al. PD-1 blockade induces responses by inhibiting adaptive immune resistance. Nature 2014; 515:568-571.

16 Topalian SL, Hodi FS, Brahmer JR, et al. Safety, activity, and immune correlates of anti-PD-1 antibody in cancer. $N$ Engl $J$ Med 2012; 366:2443-2454.

17 Topalian SL, Sznol M, McDermott DF, et al. Survival, durable tumor remission, and long-term safety in patients with advanced melanoma receiving nivolumab. J Clin Oncol 2014; 32:1020-1030.

18 Garon EB, Rizvi NA, Hui R, et al. Pembrolizumab for the treatment of non-small-cell lung cancer. N Engl J Med 2015; 372:2018-2028.

19 Borghaei H, Paz-Ares L, Horn L, et al. Nivolumab versus docetaxel in advanced nonsquamous non-small-cell lung cancer. $N$ Engl J Med 2015; 373:1627-1639.

20 Motzer RJ, Escudier B, McDermott DF, et al. Nivolumab versus everolimus in advanced renal-cell carcinoma. $N$ Engl $J$ 
Med 2015; 373:1803-1813.

21 Ansell SM, Lesokhin AM, Borrello I, et al. PD-1 blockade with nivolumab in relapsed or refractory Hodgkin's lymphoma. N Engl J Med 2015; 372:311-319.

22 Powles T, Eder JP, Fine GD, et al. MPDL3280A (anti-PD-L1) treatment leads to clinical activity in metastatic bladder cancer. Nature 2014; 515:558-562.

23 Harlin H, Meng Y, Peterson AC, et al. Chemokine expression in melanoma metastases associated with CD8+ T-cell recruitment. Cancer Res 2009; 69:3077-3085.

24 Mikucki ME, Fisher DT, Matsuzaki J, et al. Non-redundant requirement for CXCR3 signalling during tumoricidal T-cell trafficking across tumour vascular checkpoints. Nat Commun 2015; 6:7458.

25 Uze G, Schreiber G, Piehler J, Pellegrini S. The receptor of the type I interferon family. Curr Top Microbiol Immunol 2007; 316:71-95.

26 Gresser I, Belardelli F, Maury C, Maunoury MT, Tovey MG. Injection of mice with antibody to interferon enhances the growth of transplantable murine tumors. J Exp Med 1983; 158:2095-2107.

27 Gresser I, Bandu MT, Brouty-Boye D. Interferon and cell division. IX. Interferon-resistant L1210 cells: characteristics and origin. J Natl Cancer Inst 1974; 52:553-559.

28 Affabris E, Romeo G, Federico M, et al. Molecular mechanisms of action of interferons in the Friend virus-induced leukemia cell system. Haematologica 1987; 72:76-78.

29 Gresser I, Bourali C. Antitumor effects of interferon preparations in mice. J Natl Cancer Inst 1970; 45:365-376.

30 Dunn GP, Bruce AT, Sheehan KC, et al. A critical function for type I interferons in cancer immunoediting. Nat Immunol 2005; 6:722-729.

31 Fuertes MB, Kacha AK, Kline J, et al. Host type I IFN signals are required for antitumor $\mathrm{CD} 8+\mathrm{T}$ cell responses through CD8+ dendritic cells. J Exp Med 2011; 208:2005-2016.

32 Diamond MS, Kinder M, Matsushita H, et al. Type I interferon is selectively required by dendritic cells for immune rejection of tumors. J Exp Med 2011; 208:1989-2003.

33 den Haan JM, Lehar SM, Bevan MJ. CD8(+) but not CD8 (-) dendritic cells cross-prime cytotoxic T cells in vivo. J Exp Med 2000; 192:1685-1696.

34 Pooley JL, Heath WR, Shortman K. Cutting edge: intravenous soluble antigen is presented to CD4 T cells by CD8- dendritic cells, but cross-presented to CD8 T cells by CD8+ dendritic cells. J Immunol 2001; 166:5327-5330.

35 Edelson BT, Kc W, Juang R, et al. Peripheral CD103+ dendritic cells form a unified subset developmentally related to CD8 $\alpha+$ conventional dendritic cells. J Exp Med 2010; 207:823-836.

36 del Rio ML, Bernhardt G, Rodriguez-Barbosa JI, Forster R. Development and functional specialization of CD103+ dendritic cells. Immunol Rev 2010; 234:268-281.

37 Schiavoni G, Mattei F, Sestili P, et al. ICSBP is essential for the development of mouse type I interferon-producing cells and for the generation and activation of CD8(+) dendritic cells. J Exp Med 2002; 196:1415-1425.

38 Aliberti J, Schulz O, Pennington DJ, et al. Essential role for ICSBP in the in vivo development of murine CD $8 \alpha+$ dendritic cells. Blood 2003; 101:305-310.
39 Hildner K, Edelson BT, Purtha WE, et al. Batf3 deficiency reveals a critical role for $\mathrm{CD} 8 \alpha+$ dendritic cells in cytotoxic $\mathrm{T}$ cell immunity. Science 2008; 322:1097-1100.

40 Tussiwand R, Lee WL, Murphy TL, et al. Compensatory dendritic cell development mediated by BATF-IRF interactions. Nature 2012; 490:502-507.

41 Robbins SH, Walzer T, Dembele D, et al. Novel insights into the relationships between dendritic cell subsets in human and mouse revealed by genome-wide expression profiling. $\mathrm{Ge}$ nome Biol 2008; 9:R17.

42 Sancho D, Joffre OP, Keller AM, et al. Identification of a dendritic cell receptor that couples sensing of necrosis to immunity. Nature 2009; 458:899-903.

43 Maldonado-Lopez R, De Smedt T, Michel P, et al. CD8 $\alpha+$ and $\mathrm{CD} 8 \alpha$ - subclasses of dendritic cells direct the development of distinct T helper cells in vivo. J Exp Med 1999; 189:587-592.

44 Mashayekhi M, Sandau MM, Dunay IR, et al. CD $8 \alpha(+)$ dendritic cells are the critical source of interleukin-12 that controls acute infection by Toxoplasma gondii tachyzoites. Immunity 2011; 35:249-259.

45 Woo SR, Fuertes MB, Corrales L, et al. STING-dependent cytosolic DNA sensing mediates innate immune recognition of immunogenic tumors. Immunity 2014; 41:830-842.

46 Salmon H, Idoyaga J, Rahman A, et al. Expansion and activation of $\mathrm{CD} 103(+)$ dendritic cell progenitors at the tumor site enhances tumor responses to therapeutic PD-L1 and BRAF inhibition. Immunity 2016; 44:924-938.

47 Headley MB, Bins A, Nip A, et al. Visualization of immediate immune responses to pioneer metastatic cells in the lung. $\mathrm{Na}$ ture 2016; 531:513-517.

48 Thompson ED, Enriquez HL, Fu YX, Engelhard VH. Tumor masses support naive $\mathrm{T}$ cell infiltration, activation, and differentiation into effectors. J Exp Med 2010; 207:1791-1804.

49 Peske JD, Thompson ED, Gemta L, Baylis RA, Fu YX, Engelhard VH. Effector lymphocyte-induced lymph nodelike vasculature enables naive T-cell entry into tumours and enhanced anti-tumour immunity. Nat Commun 2015; 6:7114.

50 Matzinger P. An innate sense of danger. Ann NY Acad Sci 2002; 961:341-342.

51 Demaria O, De Gassart A, Coso S, et al. STING activation of tumor endothelial cells initiates spontaneous and therapeutic antitumor immunity. Proc Natl Acad Sci USA 2015; 112:15408-15413.

52 Lister MF, Sharkey J, Sawatzky DA, et al. The role of the purinergic P2X7 receptor in inflammation. J Inflamm 2007; 4:5.

53 Klarquist J, Hennies CM, Lehn MA, Reboulet RA, Feau S, Janssen EM. STING-mediated DNA sensing promotes antitumor and autoimmune responses to dying cells. J Immunol 2014; 193:6124-6134.

54 Ahn J, Konno H, Barber GN. Diverse roles of STING-dependent signaling on the development of cancer. Oncogene 2015; 34:5302-5308.

55 Huber S, Gagliani N, Zenewicz LA, et al. IL-22BP is regulated by the inflammasome and modulates tumorigenesis in the intestine. Nature 2012; 491:259-263.

56 Salcedo R, Worschech A, Cardone M, et al. MyD88-mediated signaling prevents development of adenocarcinomas of the colon: role of interleukin 18. J Exp Med 2010; 207:16251636 . 
57 Ohkuri T, Ghosh A, Kosaka A, et al. STING contributes to antiglioma immunity via triggering type I IFN signals in the tumor microenvironment. Cancer Immunol Res 2014; 2:11991208.

58 Sun L, Wu J, Du F, Chen X, Chen ZJ. Cyclic GMP-AMP synthase is a cytosolic DNA sensor that activates the type I interferon pathway. Science 2013; 339:786-791.

59 Zhang X, Shi H, Wu J, et al. Cyclic GMP-AMP containing mixed phosphodiester linkages is an endogenous high-affinity ligand for STING. Mol Cell 2013; 51:226-235.

60 Ishikawa H, Ma Z, Barber GN. STING regulates intracellular DNA-mediated, type I interferon-dependent innate immunity. Nature 2009; 461:788-792.

61 Liu S, Cai X, Wu J, et al. Phosphorylation of innate immune adaptor proteins MAVS, STING, and TRIF induces IRF3 activation. Science 2015; 347:aaa2630.

62 Ablasser A, Schmid-Burgk JL, Hemmerling I, et al. Cell intrinsic immunity spreads to bystander cells via the intercellular transfer of cGAMP. Nature 2013; 503:530-534.

63 Chen Q, Boire A, Jin X, et al. Carcinoma-astrocyte gap junctions promote brain metastasis by cGAMP transfer. Nature 2016; 533:493-498.

64 Ahn J, Xia T, Konno H, Konno K, Ruiz P, Barber GN. Inflammation-driven carcinogenesis is mediated through STING. Nat Commun 2014; 5:5166.

65 Lemos H, Mohamed E, Huang L, et al. STING promotes the growth of tumors characterized by low antigenicity via IDO activation. Cancer Res 2016; 76:2076-2081.

66 Huang L, Li L, Lemos H, et al. Cutting edge: DNA sensing via the STING adaptor in myeloid dendritic cells induces potent tolerogenic responses. J Immunol 2013; 191:3509-3513.

67 Scaffidi P, Misteli T, Bianchi ME. Release of chromatin protein HMGB1 by necrotic cells triggers inflammation. Nature 2002; 418:191-195.

68 Apetoh L, Ghiringhelli F, Tesniere A, et al. Toll-like receptor 4-dependent contribution of the immune system to anticancer chemotherapy and radiotherapy. Nat Med 2007; 13:10501059.

69 Chiba S, Baghdadi M, Akiba H, et al. Tumor-infiltrating DCs suppress nucleic acid-mediated innate immune responses through interactions between the receptor TIM-3 and the alarmin HMGB1. Nat Immunol 2012; 13:832-842.

70 Venereau E, Casalgrandi M, Schiraldi M, et al. Mutually exclusive redox forms of HMGB1 promote cell recruitment or proinflammatory cytokine release. J Exp Med 2012; 209:1519-1528.

71 Yang H, Lundback P, Ottosson L, et al. Redox modification of cysteine residues regulates the cytokine activity of high mobility group box-1 (HMGB1). Mol Med 2012; 18:250-259.

72 Rouhiainen A, Tumova S, Valmu L, Kalkkinen N, Rauvala H. Pivotal advance: analysis of proinflammatory activity of highly purified eukaryotic recombinant HMGB1 (amphoterin). J Leukoc Biol 2007; 81:49-58.

73 Beavis PA, Stagg J, Darcy PK, Smyth MJ. CD73: a potent suppressor of antitumor immune responses. Trends Immunol 2012; 33:231-237.

74 Fabbri M, Paone A, Calore F, et al. MicroRNAs bind to tolllike receptors to induce prometastatic inflammatory response. Proc Natl Acad Sci USA 2012; 109:E2110-E2116.
75 Crow YJ, Hayward BE, Parmar R, et al. Mutations in the gene encoding the 3'-5' DNA exonuclease TREX1 cause Aicardi-Goutieres syndrome at the AGS1 locus. Nat Genet 2006; 38:917-920.

76 Lee-Kirsch MA, Gong M, Chowdhury D, et al. Mutations in the gene encoding the 3'-5' DNA exonuclease TREX1 are associated with systemic lupus erythematosus. Nat Genet 2007; 39:1065-1067.

77 Ahn J, Gutman D, Saijo S, Barber GN. STING manifests self DNA-dependent inflammatory disease. Proc Natl Acad Sci USA 2012; 109:19386-19391.

78 Konno H, Konno K, Barber GN. Cyclic dinucleotides trigger ULK1 (ATG1) phosphorylation of STING to prevent sustained innate immune signaling. Cell 2013; 155:688-698.

79 Schroder K, Tschopp J. The inflammasomes. Cell 2010; 140:821-832.

80 Jorgensen I, Miao EA. Pyroptotic cell death defends against intracellular pathogens. Immunol Rev 2015; 265:130-142.

81 Corrales L, Woo SR, Williams JB, McWhirter SM, Dubensky TW Jr, Gajewski TF. Antagonism of the STING pathway via activation of the AIM2 inflammasome by intracellular DNA. J Immunol 2016; 196:3191-3198.

82 Krasnova Y, Putz EM, Smyth MJ, Souza-Fonseca-Guimaraes F. Bench to bedside: NK cells and control of metastasis. Clin Immunol 2015; pii:S1521-6616(15)30050-4.

83 Iraolagoitia XL, Spallanzani RG, Torres NI, et al. NK cells restrain spontaneous antitumor $\mathrm{CD} 8+\mathrm{T}$ cell priming through PD-1/PD-L1 interactions with dendritic cells. J Immunol 2016; 197:953-961.

84 Peng G, Wang HY, Peng W, Kiniwa Y, Seo KH, Wang RF. Tumor-infiltrating gammadelta $\mathrm{T}$ cells suppress $\mathrm{T}$ and dendritic cell function via mechanisms controlled by a unique toll-like receptor signaling pathway. Immunity 2007; 27:334-348.

85 Robertson FC, Berzofsky JA, Terabe M. NKT cell networks in the regulation of tumor immunity. Front Immunol 2014; 5:543.

86 Dadi S, Chhangawala S, Whitlock BM, et al. Cancer immunosurveillance by tissue-resident innate lymphoid cells and innate-like T cells. Cell 2016; 164:365-377.

87 Lee YK, Mazmanian SK. Has the microbiota played a critical role in the evolution of the adaptive immune system? Science 2010; 330:1768-1773.

88 Hooper LV, Littman DR, Macpherson AJ. Interactions between the microbiota and the immune system. Science 2012; 336:1268-1273.

89 Ivanov, II, McKenzie BS, Zhou L, et al. The orphan nuclear receptor RORgammat directs the differentiation program of proinflammatory IL-17 $7^{+}$T helper cells. Cell 2006; 126:11211133.

90 Sivan A, Corrales L, Hubert N, et al. Commensal Bifidobacterium promotes antitumor immunity and facilitates anti-PD-L1 efficacy. Science 2015; 350:1084-1089.

91 Kawahara T, Takahashi T, Oishi K, et al. Consecutive oral administration of Bifidobacterium longum MM-2 improves the defense system against influenza virus infection by enhancing natural killer cell activity in a murine model. Microbiol Immunol 2015; 59:1-12.

92 Ganal SC, Sanos SL, Kallfass C, et al. Priming of natural killer cells by nonmucosal mononuclear phagocytes requires in- 
structive signals from commensal microbiota. Immunity 2012; 37:171-186.

93 Abt MC, Osborne LC, Monticelli LA, et al. Commensal bacteria calibrate the activation threshold of innate antiviral immunity. Immunity 2012; 37:158-170.

94 Vetizou M, Pitt JM, Daillere R, et al. Anticancer immunotherapy by CTLA-4 blockade relies on the gut microbiota. Science 2015; 350:1079-1084.

95 Iida N, Dzutsev A, Stewart CA, et al. Commensal bacteria control cancer response to therapy by modulating the tumor microenvironment. Science 2013; 342:967-970.

96 Viaud S, Saccheri F, Mignot G, et al. The intestinal microbiota modulates the anticancer immune effects of cyclophosphamide. Science 2013; 342:971-976.

97 Gajewski TF, Schreiber H, Fu YX. Innate and adaptive immune cells in the tumor microenvironment. Nat Immunol 2013; 14:1014-1022.

98 Salerno EP, Olson WC, McSkimming C, Shea S, Slingluff CL Jr. T cells in the human metastatic melanoma microenvironment express site-specific homing receptors and retention integrins. Int J Cancer 2014; 134:563-574.

99 Erdag G, Schaefer JT, Smolkin ME, et al. Immunotype and immunohistologic characteristics of tumor-infiltrating immune cells are associated with clinical outcome in metastatic melanoma. Cancer Res 2012; 72:1070-1080.

100 Ribas A, Robert C, Hodi S, et al. Association of response to programmed death receptor 1 (PD-1) blockade with pembrolizumab (MK-3475) with an interferon-inflammatory immune gene signature. J Clin Oncol 2015; 33:(suppl; abstr 3001).

101 Seiwert TY, Burtness B, Weiss J, et al. Inflamed-phenotype gene expression signatures to predict benefit from the anti-PD-1 antibody pembrolizumab in PD-L1+ head and neck cancer patients. J Clin Oncol 2015; 33:(suppl; abstr 6017).

102 Spranger S, Bao RY, Gajewski TF. Melanoma-intrinsic beta-catenin signalling prevents anti-tumour immunity. Nature 2015; 523:231-U261.

103 Conlon J, Burdette DL, Sharma S, et al. Mouse, but not human STING, binds and signals in response to the vascular disrupting agent 5,6-dimethylxanthenone-4-acetic acid. $J$ Immunol 2013; 190:5216-5225.

104 Gao P, Ascano M, Zillinger T, et al. Structure-function analysis of STING activation by $\mathrm{c}\left[\mathrm{G}\left(2^{\prime}, 5^{\prime}\right) \mathrm{pA}\left(3^{\prime}, 5^{\prime}\right) \mathrm{p}\right]$ and targeting by antiviral DMXAA. Cell 2013; 154:748-762.

105 Kim S, Li L, Maliga Z, Yin Q, Wu H, Mitchison TJ. Anticancer flavonoids are mouse-selective STING agonists. ACS Chem Biol 2013; 8:1396-1401.

106 Prantner D, Perkins DJ, Lai W, et al. 5,6-Dimethylxanthenone-4-acetic acid (DMXAA) activates stimulator of interferon gene (STING)-dependent innate immune pathways and is regulated by mitochondrial membrane potential. $J$ Biol Chem 2012; 287:39776-39788.

107 Corrales L, Glickman LH, McWhirter SM, et al. Direct activation of STING in the tumor microenvironment leads to potent and systemic tumor regression and immunity. Cell Rep 2015; 11:1018-1030.

108 Lara PN Jr, Douillard JY, Nakagawa K, et al. Randomized phase III placebo-controlled trial of carboplatin and paclitaxel with or without the vascular disrupting agent vadimezan (ASA404) in advanced non-small-cell lung cancer. J Clin On- col 2011; 29:2965-2971.

109 Aduro Biotech Inc, Novartis Pharmaceuticals. Study of the Safety and Efficacy of MIW815 (ADU-S100) in Patients with Advanced/Metastatic Solid Tumors or Lymphomas. 2020. ClinicalTrials.gov [Internet]. Bethesda (MD): National Library of Medicine (US). Identifier: NCT02675439. Available from: https:/ClinicalTrials.gov/show/NCT02675439. (cited 01 July 2016).

110 Brody JD, Ai WZ, Czerwinski DK, et al. In situ vaccination with a TLR9 agonist induces systemic lymphoma regression: a phase I/II study. J Clin Oncol 2010; 28:4324-4332.

111 Caskey M, Lefebvre F, Filali-Mouhim A, et al. Synthetic double-stranded RNA induces innate immune responses similar to a live viral vaccine in humans. $J$ Exp Med 2011; 208:23572366.

112 Dowling DJ, Tan Z, Prokopowicz ZM, et al. The ultra-potent and selective TLR8 agonist VTX-294 activates human newborn and adult leukocytes. PLoS One 2013; 8:e58164.

113 Dynavax Technologies Corporation. Study of SD-101 in Combination with Localized Low-dose Radiation in Patients with Untreated Low-grade B-cell Lymphoma. 2016. ClinicalTrials.gov [Internet]. Bethesda (MD): National Library of Medicine (US). Identifier: NCT02266147. Available from: https://ClinicalTrials.gov/show/NCT02266147. (cited 01 Jul 2016).

114 Immune Design, Merck Sharp \& Dohme Corp. Study of Intratumoral G100 with or without Pembrolizumab in Patients with Follicular Non-Hodgkin's Lymphoma. 2017. ClinicalTrials.gov [Internet]. Bethesda (MD): National Library of Medicine (US). Identifier: NCT02501473. Available from: https:// ClinicalTrials.gov/show/NCT02501473. (cited 01 July 2016).

115 MedImmune LLC. A Study of MEDI9197 Administered in Subjects with a Solid Tumor Cancer. 2018. ClinicalTrials.gov [Internet]. Bethesda (MD): National Library of Medicine (US). Identifier: NCT02556463. Available from: https://ClinicalTrials.gov/show/NCT02556463. (cited 01 Jul 2016).

116 Oncovir Inc, National Institutes of Health, Icahn School of Medicine at Mount Sinai, Bay Hematology Oncology, Emory University, University of Pittsburgh, National Cancer Institute. In Situ, Autologous Therapeutic Vaccination Against Solid Cancers with Intratumoral Hiltonol®. 2018. ClinicalTrials.gov [Internet]. Bethesda (MD): National Library of Medicine (US). Identifier: NCT02423863. Available from: https:// ClinicalTrials.gov/show/NCT02423863. (cited 01 Jul 2016).

117 Deng L, Liang H, Xu M, et al. STING-dependent cytosolic DNA sensing promotes radiation-induced type I interferon-dependent antitumor immunity in immunogenic tumors. Immunity 2014; 41:843-852.

118 University of Chicago. Study of PD1 Blockade by Pembrolizumab with Stereotactic Body Radiotherapy in Advanced Solid Tumors. 2017. ClinicalTrials.gov [Internet]. Bethesda (MD): National Library of Medicine (US). Identifier: NCT02608385. Available from: https://ClinicalTrials.gov/ show/NCT02608385. (cited 01 July 2016).

119 Luke JJ, Bao R, Spranger S, Sweis RF, Gajewski T. Correlation of $\mathrm{WNT} / \beta$-catenin pathway activation with immune exclusion across most human cancers. J Clin Oncol 2016; 34:(suppl; abstr 3004).

120 Peng W, Chen JQ, Liu C, et al. Loss of PTEN promotes re- 
sistance to T cell-mediated immunotherapy. Cancer Discov 2016; 6:202-216.

121 Sweis RF, Spranger S, Bao R, et al. Molecular drivers of the non-T-cell-inflamed tumor microenvironment in urothelial bladder cancer. Cancer Immunol Res 2016; 4:563-568.

122 Casey SC, Tong L, Li Y, et al. MYC regulates the antitumor immune response through CD47 and PD-L1. Science 2016; 352:227-231. 